\title{
EL PROCESO DE MODERNIZACIÓN DEL ESTADO PERUANO: AS- PECTOS IMPORTANTES A TENER EN CUENTA EN LA GESTIÓN PÚBLICA
}

Christian A. Romero Hidalgo ${ }^{1}$

El proceso de modernización del Estado Peruano declarado por la Ley $N^{\circ} 27658$, tiene como finalidad primordial mejorar la gestión pública y construir un Estado democrático, descentralizado y al servicio del ciudadano. Dicho proceso busca obtener mayores niveles de eficiencia del aparato estatal, de manera que se logre una mejor atención a la ciudadanía, priorizando y optimizando el uso de los recursos públicos; es por ello que mediante un adecuado uso y relación del Gobierno electrónico con el estado se podrá lograr la tan ansiada eficiencia y eficacia del aparato estatal. Respecto a los temas de gobierno electrónico y gestión pública se han encontrado diversas investigaciones en diferentes ámbitos. En el ámbito internacional:

Respecto a la modernización del gobierno desde el punto de vista de las nuevas TIC, está dada por la innovación que a través del gobierno electrónico están consiguiendo las ciudades, los estados y los países. (Aguilar, 2006). Esta modernización tiene por objeto maximizar la eficiencia, reducir costos guberna- mentales y cambiar dentro de lo factible la forma como se implementan algunos programas de gobierno. La innovación consiste en la introducción de novedades en las técnicas de operación del suministro de servicios y en las formas de organización de la toma de decisiones y la coordinación del trabajo conjunto que desempaña cualquier órgano de la administración pública. Esto plantea una transformación paulatina del gobierno.

En cuanto al Gobierno electrónico se encuentran en el documento Política Nacional de Informática. La Política en cuestión tiene cuatro grandes propósitos estratégicos, que permiten englobar las líneas de acción necesarias para: a) la incorporación ordenada, racional, sistemática y efectiva de las TICs en el país, b) la organización de los servicios del gobierno electrónico, y c) la construcción de las bases y acuerdos que permitan facilitar el tránsito hacia la Sociedad Global de la Información y del Conocimiento. Estos propósitos son:

Propósito 1: Modernizar la gestión pública y propiciar la descentralización del Estado, mediante el uso intensivo de las tecnologías de información.

Propósito 2: Promover el aumento de la productividad en la Administración Pública, empresas y ciudadanos, por medio del uso intensivo de las tecnologías de Información.

Propósito 3: Promover el acceso universal a la información y el conocimiento.

Propósito 4: Promover la Sociedad de la Información y Conocimiento.

El Propósito 1 es el que plantea como objetivo implementar un nuevo modelo de gestión en la Administración Pública, modelo denominado "Gobierno Electrónico". Para lograr este objetivo se enuncian los siguientes lineamientos estratégicos: a. Construir una plataforma tecnológica para la modernización de la gestión pública y la descentralización. b. Impulsar la implantación de un modelo de gestión apoyado por las tecnologías de información para la Administración Pública. c. Impulsar el desarrollo e implantación de servicios públicos abiertos y en línea.

Doctorando en Derecho por la Universidad Nacional de Trujillo. Magister en Gestión Pública por la Universidad Cesar Vallejo. Director del Programa de Derecho de la Universidad Cesar Vallejo filiales de Chimbote y Huaraz. Docente Universitario. 
Para lograr todos esos objetivos era necesario tener un adecuado marco legal a fin de poder desarrollar y aplicar el gobierno electrónico en el Perú, dichas normas son:

- Ley $\mathrm{N}^{\circ} 27269$ de Firmas y certificados digitales, promulgada el 15/07/2000, y su Reglamento, D. S. $\mathrm{N}^{\circ}$ 019-2002-JUS del 15/05/2002. Permite la celebración de contratos por medios electrónicos, plantea la ley de firma digital y la regulación del registro de nombres de dominio en el Perú.

- Ley $N^{\circ} 27310$, promulgada el 15/07/2000. Modifica el Artículo 11 de la Ley $N^{\circ}$ 27269 , disponiendo que los certificados de firmas digitales emitidos por entidades extranjeras tendrán la misma validez y eficacia jurídica reconocidas en la Ley, siempre y cuando tales certificados sean reconocidos por la autoridad administrativa competente.

- Ley $\mathrm{N}^{\circ} 27291$, promulgada el 24/06/2000. Modifica el Código Civil permitiendo la utilización de los medios electrónicos para la comunicación de la manifestación de la voluntad y la utilización de la firma electrónica.

- Ley $N^{\circ} 27419$, promulgada el 02/07/2001. Sobre notificación por correo electrónico (permite el uso del correo electrónico y otros medios para la notificación en el ámbito del Código Procesal Civil).
- Resolución N N 103-2003 -CRT-INDECOPI de la Comisión de Reglamentos Técnicos y Comerciales, promulgada el 15/11/2003. Aprueba disposiciones complementarias al reglamento de la Ley de Firmas y Certificados Digitales.

- Decreto Legislativo $N^{\circ}$ 681, promulgado el $11 / 10 / 1991$, modificatorias, y su reglamento D.S. 009-92 JUS. Dicta normas que regulan el uso de tecnologías avanzadas en materia de archivo de documentos e información, tanto respecto a la elaborada en forma convencional cuanto la producida por procedimientos informáticos. Sobre los efectos legales de los documentos digitales obtenidos producto del microfilmado.

- Decreto Supremo 0182001-PCM, publicado el 27/02/2001. Dispone que Entidades del Sector Público incorporen en su Texto Único de Procedimientos Administrativos (TUPA) procedimientos para facilitar el acceso a la información. Declara que esos procedimientos debe realizarse utilizando medios electrónicos.

- Decreto de Urgencia $N^{\circ}$ 077-2001, publicado el 07/07/2001. Crea el portal de transparencia económica del Ministerio de Economía y Finanzas, que sirve para seguimiento ciudadano de uso de los recursos del tesoro por parte de las instituciones públicas.

- Ley 27444 del Procedimien- to Administrativo General, promulgada el 10/04/2001. Permite a las administraciones públicas la utilización de medios electrónicos en el marco de su actuación administrativa, y el envío de notificaciones y de documentación a los administrados mediante correo electrónico o cualquier otro medio de transmisión de datos.

- Ley N² 27806 Transparencia y acceso a la información pública, promulgada el $02 / 08 / 2002$. Tiene por finalidad promover la transparencia de los actos del Estado y regular el derecho fundamental del acceso a la información consagrado en el numeral 5 del Artículo $2^{\circ}$ de la Constitución Política del Perú. Se modificó mediante Ley 27972 y se reglamentó mediante D.S. $\mathrm{N}^{\circ}$ 072-2003-PCM.

De todo lo expuesto, concluimos sosteniendo que el Gobierno Electrónico se podría ver como un paso más allá en el proceso de reforma de la gestión pública.

Aun cuando los beneficios que puede brindar la implementación de un Gobierno Electrónico a todos los niveles de la sociedad son enormes, se pueden encontrar rechazos y reticencias especialmente al interior de los estamentos públicos. Esto debido a que este nuevo paradigma trae consigo un cambio en la manera de pensar, de actuar y de compartir la información. Se requiere hacer reestructuración de procesos, innovación en los servicios, utilización de 
nuevas tecnologías, etc. Esto provoca resistencias, temores al uso de las nuevas tecnologías, inseguridades al asociar tecnología con pérdida de puestos de trabajo, rechazo a capacitarse en áreas nuevas, etc. A menudo este cambio se percibe por parte de algunos funcionarios como una pérdida de poder, especialmente cuando éste se ha construido sobre la base de haberse apropiado de información que ahora será de amplio acceso y compartida por todos.

Beneficios como mejoramiento de la calidad en la prestación de servicios públicos, eficiencia, eficacia, y transparencia, entre otros, son los beneficios más tangibles como resultado de la implementación de un Gobierno Electrónico.

Una forma útil de presentar estos beneficios es entendiéndolos como resultados de ciertas acciones e iniciativas de gobierno electrónico, en otras palabras, cómo los proyectos que harán posible materializar estos cambios entregarán beneficios a la ciudadanía. En este sentido, los beneficios podrían resumirse de la siguiente manera: Mejoramiento de la calidad de los servicios públicos, eficiencia y productividad en los procesos y operación gubernamental, transparencia y rendición de cuentas, participación ciudadana, marco regulatorio que respalde el gobierno electrónico, marco legal y regulatorio que fomente la sociedad de la información, y burocracia en el sector público y concepto de ventanilla única electrónica del Estado.

Ruelas y Pérez (2006) señalan que El gobierno electrónico o e-gobierno también llamado gobierno en línea o gobierno digital es un concepto que engloba a todas aquellas actividades basadas en las modernas tecnologías de la información y la comunicación (en particular, Internet) que el Estado desarrolla para aumentar la eficiencia de la gestión pública, mejorar los servicios ofrecidos a los ciudadanos y proveer las acciones de gobierno en un marco mucho más transparente que el actual. Digitalizar el gobierno no es solo instalar unas cuantas computadoras o diseñar una página web que ofrezca información, sino que supone transformar la relación fundamental que existe entre el gobierno y los ciudadanos.

El gobierno electrónico se refiere a la continua optimización de los servicios del gobierno, la participación ciudadana y gobernación mediante la transformación de las relaciones internas y externas a través de la tecnología y la Internet. Este medio puede aumentar la eficiencia y transparencia de la administración pública, así como incrementar la capacidad institucional de los gobiernos locales, regionales y nacionales (Carrillo, 2006)

De otro lado, cabe precisar que transparencia, El término transparencia deriva del latín 'trans' (más allá de, a través de) y de 'parere' (aparecer, mostrar o mostrarse).

En sentido propio, la transparencia es el carácter o la propiedad de algo que es transparente, o sea, algo que se deja atravesar por la luz o que per- mite el paso de la luz, dejando ver formas y colores.

En sentido figurado, el término "transparencia" es utilizado para caracterizar una práctica social guiada por la sinceridad y por la perfecta o casi perfecta accesibilidad a toda la información vinculada, y que concierne e interesa a la opinión pública, o a un sector de la misma, o incluso a un solo individuo. El objetivo primero de la transparencia, es de establecer una relación de confianza entre quien pide o exige la transparencia y quien la da, y se opone al concepto de opacidad.

En el marco de la administración pública, la transparencia es un mecanismo que previene actos de corrupción y que permite a la ciudadanía conocer el funcionamiento interno de las instituciones y cómo se manejan los fondos que éstas reciben

Según Delpiazzo (2009), una característica esencial de todo Estado Democrático y Constitucional es la publicidad de sus actos y la transparencia de la administración estatal en la gestión de los asuntos públicos. Ello implica que los funcionarios públicos rindan cuentas sobre las decisiones que adoptan y que las personas puedan solicitar y acceder a la información completa y veraz que debe obrar en las entidades públicas.

De esta manera, la transparencia en la gestión pública contribuye a prevenir la corrupción, fortalecer el vínculo entre el Estado y la pobla- 
ción, y promover la vigilancia ciudadana.

La transparencia es uno de los temas más filosóficos y subjetivos que podemos tratar, ya que se basa en valores éticos, y aunado a ello, en el valor agregado que todo servidor público debe aplicar en el ejercicio de su función, de ahí la necesidad de detenernos en su análisis y en su confrontación con otros conceptos tales como el derecho a la información, el acceso a la información y la rendición de cuentas con los que la transparencia convive y se interrelaciona diariamente en la labor pública.

Pudiéramos pensar en que el término transparencia es sinónimo de derecho de acceso a la información o de rendición de cuentas lo cual no es del todo correcto pues, aunque es cierto que mantienen relación entre sí, son conceptos completamente diferentes, y esto va más allá de los números que nos presentan. El acceso a la información designa a aquella información que debe solicitarse para obtenerla, por lo tanto, es el ejercicio libre de una garantía constitucional por medio del cual la sociedad pueden: conocer, solicitar y supervisar las acciones de su propio gobierno, y por consiguiente la información que nos proporcionan debe ser de dominio público

Transparencia en la gestión pública, en un sentido amplio, la transparencia implicaría una "política de transparencia", en tanto que "acceso a la información", se entendería como "el derecho de acceso a la información", siendo éste último un instrumento de la transparencia

Según Mauricio Merino “El derecho de acceso a la información pública es, en la práctica, el derecho que tienen las personas para conocer los documentos que producen $\mathrm{y}$ manejan las autoridades públicas" y que por lo tanto, este conocimiento puede llegar a ser un verdadero motor político de transparencia social, o sea, que esto implica que para poder movilizar todo el engranaje que se necesita para que la información sea pública, se debe de destinar otro porcentaje del PIB para que el derecho de acceso a la información pública, sea en un principio la prerrogativa de toda persona para acceder y examinar datos y registros públicos en poder de los sujetos obligados, y también saber de aquello que el Estado considera nocivo para entregar la posibilidad de revisar y analizar dicha información.

La transparencia es el resultado de una forma de gobernar, de administrar y de gestionar al Estado, de lo cual se deduce que este concepto es un valor de la democracia. La transparencia tiene que ver con el desempeño de la comunidad política, de sus representantes así como también de sus autoridades quienes tienen que aclarar sus acciones para fortalecer al gobierno y su gestión pública, como consecuencia.

Los procesos de toma de decisión y verificación en la gestión política administrativa del Estado proponiendo la colaboración de la transparencia como un instrumento político para su aplicación y la construcción de una sistematización para la apropiación de la auditoría social, así mismo como una herramienta política de la sociedad civil. Un ciudadano como tú y como yo, en pleno derecho facultativo y en pleno ejercicio de nuestras funciones elegidas, podemos garantizar la probidad, eficiencia y eficacia de la administración pública.

Información fidedigna, en Internet hay cientos de millones de páginas web con una gran variedad y cantidad de información. Esta información es dinámica y volátil: a diferencia de otras tecnologías, Internet permite que la información se modifique en cualquier momento.

A través de las páginas web podemos acceder a información en diferentes formatos y soportes, tales como textos, gráficos, imágenes, sonidos, videos, presentaciones multimediales, etcétera.

La cantidad y variedad de información disponible en Internet determina la necesidad de contar con ciertas herramientas para obtener información que resulte significativa, es decir, útil, relevante y confiable. Para ello es necesario que al iniciar un proceso de búsqueda se consideren los siguientes aspectos:

- Conocimiento de los recursos involucrados: es decir, características de la red, programas de navegación, de administración de archivos y otras utilidades. 
- Conocimiento de los sitios de búsqueda y sus respectivas estrategias de búsqueda.

La información es fidedigna cuando adquiere valor en la medida que sirve para tomar decisiones, es útil y suficiente, actualizada frecuentemente, se localiza con rapidez, es segura.

Asimismo, el derecho de acceso a la información pública implica la concreción del derecho a la libertad de expresión, entendido éste en toda su amplitud y extendido a lo que hoy llamamos Derecho a la Información, que comprende tanto la facultad de dar como de recibir información, y cuyo ejercicio corresponde a toda persona: al "sujeto universal", a todos y cada uno de nosotros.

A su vez, el derecho de acceso a la información guarda una ínsita relación con otros derechos humanos y contribuye a su efectivo ejercicio, tal como señala la Relatoría para la Libertad de Expresión en el ámbito de la Comisión Interamericana de Derechos Humanos en su Informe del año 2002: "En un sistema democrático representativo y participativo, la ciudadanía ejerce sus derechos constitucionales de participación política, votación, educación y asociación, entre otros, a través de una amplia libertad de expresión y de un libre acceso a la información. La publicidad de la información permite que el ciudadano pueda controlar (la gestión pública), no solo por medio de una constatación de los mismos con la ley, que los gobernantes han jurado cumplir, sino también ejerciendo el derecho de petición y de obtener una transparente rendición de cuentas.

El acceso a la información, a la vez de conformarse como un aspecto importante de la libertad de expresión, se conforma como un derecho que fomenta la autonomía de las personas, y que les permite la realización de un plan de vida

"La presencia de la democracia de un Estado se encuentra en la información que proporcionen los poderes públicos: en que esta información exista y sea fluida y suficiente $y$ en que no esté tergiversada. Por eso hay que hacer, como se ha hecho muchas veces en la doctrina, una equiparación entre totalitarismo y desinformación".

En este marco, se estima pertinente esbozar esquemáticamente algunas implicancias del derecho de acceso a la información pública, ya que este somero análisis da fe del efecto positivo y multiplicador que presenta su constante ejercicio:

- Concreción del derecho a buscar y recibir información. La correspondencia entre el deber de informar de la Administración y el derecho a la información de los ciudadanos.

- Reconocimiento y garantía de una institución política fundamental: la opinión pública libre, indisolublemente ligada con el pluralismo político. Permite que el ciudadano pueda formar libremente sus opiniones, combatir civilizadamente las ideas contrapuestas e incluso hasta ponderarlas.

- Instrumento para efectivizar el Principio de Transparencia administrativa, de gestión y de los actos de gobierno. Instrumento para la realización de un control democrático sobre la actividad administrativa.

- Fomento y garantía de participación de modo más responsable en los asuntos públicos. Instrumento necesario para una correcta formación de la voluntad democrática.

- Afianzamiento del sistema de relaciones democráticas auspiciado por la Constitución, así como el ejercicio efectivo de otros derechos y libertades.

- Derecho a la igualdad. Tratamiento común de los ciudadanos en sus relaciones con la Administración Pública.

- Debilitamiento de la línea divisoria entre gobernantes y gobernados en una democracia participativa.

- Obliga al Estado tanto a abstenerse de interferir en el acceso a la información, como de facilitarla con políticas activas.

Ciertamente, la introducción del derecho de acceso a la información pública en el sistema jurídico y particularmente en los textos constitucionales, contribuye a que los ciudadanos puedan evaluar de mejor manera el desempeño de los 
gobernantes, amplía la garantía y fundamento del derecho a la información, fortalece la democracia y otorga una herramienta concreta para la transparencia del sistema.

En relaciónalas Tecnologías dela Información y la Comunicación, Fernández (2001), las define como el conjunto de actividades de investigación, desarrollo, fabricación, integración, instalación, comercialización y mantenimiento de componentes, subconjuntos, productos y sistemas físicos y lógicos, fundamentados en la tecnología electrónica, así como la explotación de servicios basados en dichas tecnologías, la producción y difusión de contenidos soportados electrónicamente y las aplicaciones de internet.

Como instrumentos y procesos utilizados para recuperar, almacenar, organizar, manejar, producir, presentar e intercambiar información por medios electrónicos y automáticos, las TIC son aquellas tecnologías que permiten transmitir, procesar y difundir información de manera instantánea. Son consideradas la base para reducir la Brecha Digital sobre la que se tiene que construir una Sociedad de la Información y una Economía del Conocimiento.

El modelo de la nueva Gestión Pública:

La Nueva Gestión Pública busca satisfacer las necesidades de los ciudadanos a través de una gestión pública eficiente y eficaz. Para este enfoque, es imperativo el desarrollo de servicios de mayor calidad en un marco de sistemas de con- trol que permitan transparencia en los procesos de elección de planes y resultados, así como en los de participación ciudadana, la NGP es el paradigma donde se inscriben los distintos procesos de cambio en la organización y gestión de las administraciones públicas.

Es un enfoque que intenta incorporar algunos elementos de la lógica privada a las organizaciones públicas. Es decir que la NGP es una búsqueda de lograr una mayor productividad en eficiencia colectiva, porque no sólo se espera el cumplimiento de metas por parte de la responsabilidad de liderazgo de quienes la dirigen sino y fundamentalmente es cuánto hemos comprometido al ciudadano en aquel éxito.

En síntesis la Nueva Gestión Pública está fundamentada sobre: La formulación estratégica de políticas de desarrollo y gestión, la gradual eliminación del modelo burocrático hacia una GESTIÓN POR RESULTADOS, la creación del valor público, el desarrollo de las instituciones y dimensionamiento adecuado del Estado, y el mejoramiento de las conquistas macroeconómicas y la equidad social.

La OCDE (1995: 28 y 1997: 3750) considera que esta nueva forma de gestión de la Administración Pública se caracteriza por:

- Desregulación. Este planteamiento busca la disminución de reglas y normas en el Sector Público, intentando a la vez que las que existan permitan un planteamiento estratégico de la gestión a través de la flexibilidad en su aplicación.

- Descentralización de los poderes de la gestión. La idea de descentralización supone la creación de unidades (entidades, agencias, etc.) más reducidas, con flexibilidad en las normas a aplicar y abandonadas a merced del mercado.

- Énfasis en las responsabilidades de los gestores y motivación para la mejora.

- El reforzamiento de las capacidades estratégicas del centro, junto con la reorganización e implantación de la función pública en la gestión y las reformas.

- Gestión más orientada hacia el cliente.

- Laintroduccióndelacompetencia y el mercado.

- Utilización de métodos de evaluación y técnicas de gestión aplicadas en el ámbito empresarial.

Sin embargo, como se ha mostrado, existen deficiencias en la prestación de los servicios públicos, las cuales terminan incidiendo negativamente en la percepción ciudadana sobre la gestión pública y el desempeño del Estado en el Perú. Frente a ello, ¿cuáles son los principales problemas de gestión que deben ser resueltos?

- Ausencia de un sistema eficiente de planeamiento y problemas de articulación con el sistema de presupuesto público 
- Deficiente diseño de la estructura de organización y funciones

- Inadecuados procesos de producción de bienes y servicios públicos

- Infraestructura, equipamiento y gestión logística insuficiente

- Inadecuada política y gestión de recursos humanos

- Limitada evaluación de resultados e impactos, así como seguimiento y monitoreo de los insumos, procesos, productos y resultados de proyectos y actividades

- Carencia de sistemas y métodos de gestión de la información y el conocimiento

- Débil articulación intergubernamental e intersectorial

En relación a las principales características del nuevo paradigma de la gestión pública, se pueden señalar las siguientes:

- Adopción del principio del ciudadano-cliente o usuario, con derechos claramente especificados y respetados.

\section{- Definición de un nuevo} perfil de administrador, ejecutivo o gerente público, guiado por la búsqueda de resultados y evaluado en consecuencia, sujeto a la rendición de cuentas y a la competencia no solo al momento de la contratación.
- Establecimiento de contratos de gestión, que permiten explicitar la visión, misión y objetivos de las entidades ejecutoras $\mathrm{y}$, a su vez, sirven de base para la evaluación de estas, a través de la definición de indicadores de desempeño.

La visión: en un estado moderno al servicio del ciudadano, los ciudadanos demandan un Estado Moderno, al servicio de las personas, lo cual implica una transformación de sus enfoques y prácticas de gestión, concibiendo sus servicios o intervenciones como expresiones de derechos de los ciudadanos.

Con ese sentido, la presente política caracteriza un Estado Moderno como aquél orientado al ciudadano, eficiente, unitario y descentralizado, inclusivo y abierto (transparente $\mathrm{y}$ que rinde cuentas).

Orientado al ciudadano: el Estado asigna sus recursos, diseña sus procesos y define sus productos y resultados en función de las necesidades de los ciudadanos. En tal sentido, sin perder sus objetivos esenciales, es flexible para adecuarse a las distintas necesidades de la población y a los cambios sociales, políticos y económicos del entorno. Por lo tanto, se expresa en una gestión pública en la que funcionarios públicos calificados y motivados se preocupan por entender y responder a las necesidades de los ciudadanos.

Eficiente: el Estado genera mayor valor público a través de un uso racional de los recursos con los que cuenta, bus- cando proveer a los ciudadanos lo que necesitan, al menor costo posible, con un estándar de calidad adecuado y en las cantidades óptimas que maximicen el bienestar social.

Unitario y Descentralizado: el Estado busca satisfacer las necesidades de la ciudadanía adaptando sus políticas a las diferentes necesidades y condicionantes existentes en cada espacio territorial, a través de gobiernos descentralizados autónomos en su ámbito de competencias y sujetos a políticas, rectorías y normas nacionales que garanticen los derechos que corresponden a todos por igual.

Inclusivo: el Estado busca asegurar en todas sus acciones que todos los ciudadanos tengan igualdad de oportunidades en el acceso a sus servicios y en la elección de sus opciones de vida, buscando cerrar las brechas existentes. Procura brindar a todos los ciudadanos, servicios de calidad y en la cantidad necesaria para satisfacer sus necesidades.

Abierto: el Estado es transparente y accesible a los ciudadanos, fomenta la participación ciudadana, la integridad pública y rinde cuentas de su desempeño.

Un Estado Moderno con tales atributos será capaz de garantizar a todos los ciudadanos un creciente acceso a bienes y servicios públicos de calidad, de manera equitativa, oportuna y pertinente; permitiendo así reducir las brechas sociales y económicas existentes como resultado de un crecimiento desigual del país, y ejerciendo con responsabili- 
dad su rol promotor de desarrollo del país.

Con esta visión de Estado Moderno, planteamos emprender un proceso de cambio en y reforma integral de la gestión pública, a nivel gerencial y operacional, que pueda afrontar la debilidad estructural del aparato estatal para cumplir sus objetivos y así, pasar de una administración pública que se mira a sí misma, a una enfocada en la obtención de resultados para los ciudadanos. En tal sentido es que se plantea el impulso del proceso de modernización de la gestión pública, sostenido y con perspectiva de largo plazo, implicando para todas las entidades la realización de acciones orientadas a incrementar los niveles de eficiencia y eficacia en la gestión pública, de modo que ésta pueda cumplir con sus funciones institucionalmente asignadas destinadas a servir más y mejor a los ciudadanos.

En cuanto al tema de la modernización de la Gestión Pública en nuestro País, implica una Reforma del Estado misma que debe involucrar a los 3 poderes del Estado y los 3 niveles de gobierno, conducir a cambios sustanciales en la organización y distribución del poder político, en la estructura y funcionamiento del aparato estatal, en el vínculo con los trabajadores del sector público, y en la forma en la que el Estado se relaciona con los ciudadanos y ciudadanas.

Desde la Presidencia del Concejo de Ministros, a través de la Secretaría de Gestión Pública, se está iniciando el proceso de reforma del sistema administrativo, el cual se traduce en la modernización de la gestión pública que busque perfeccionar estructuras, métodos e instrumentos del Estado y esté dirigida a la ciudadanía.

Buscar un modelo de gestión pública orientada a resultados que impacte en el bienestar de los ciudadanos y ciudadanas, que sea descentralizada con respuestas diferenciadas para cada nivel de gobierno y territorio y que sea permanentemente evaluada y monitoreada.

Durante la última década, los avances más importantes en el ámbito de la reforma administrativa han sido:

- Modernización de la Gestión Pública (proceso de descentralización simplificación administrativa y mayor transparencia)

- Presupuestos por resultados (que se encuentra en su segunda fase), que requiere una modificación en la gestión de cada entidad para que logre los resultados buscados.

- Inicio de la reforma del Servicio Civil (creación de SERVIR)

Esto significa que para lograr una gestión pública moderna orientada a resultados, las entidades deben:

- Desarrollar canales para lograr mayor transparencia, accesibilidad y participación de todos los ciudadanos y rendición de cuentas por el Estado;

- Desarrollar y emplear intensivamente tecnologías de información y comunicación (TIC) que permitan acercar los servicios del Estado a los ciudadanos y empresas, y optimizar los procesos de todos y cada uno de los organismos que conforman la Administración Pública;

- Coordinar y cooperar entre sí, a fin de articular y complementar objetivos, acciones, recursos y capacidades institucionales en función de maximizar el rendimiento de dichos recursos y capacidades y a través de estos, el desempeño y los servicios del Estado a favor de los ciudadanos; y finalmente;

- Fomentar un cambio cultural en las instituciones públicas que permita sostener el esfuerzo de modernización, el cual deberá partir por entender la necesidad de cambiar la gestión pública hacia una gestión por resultados. En ese sentido, la gestión del cambio constituye el motor que hará posible sostener este salto de una gestión centrada en sí misma a una que enfoque su accionar en el ciudadano.

La gestión pública, de manera general, pretende fortalecer la capacidad administrativa y de respuesta del Estado. Frente a la preocupación por los problemas administrativos de los gobiernos, la gestión pública es una herramienta útil para mejorar sus resultados, así como su mayor nivel de aceptación (legitimidad) por parte de los ciudadanos. La innovación de modernización 
de la gestión pública busca lograr el mayor impacto posible de la acción del Estado en sus diversos niveles, en estrecha coordinación con la Sociedad Civil y sus Sectores de Organización, en la mejora de la calidad de vida de la población y la construcción de sus oportunidades de futuro (Canales, 2011).

\section{Concluyendo:}

El proceso de modernización del Estado Peruano declarado por la Ley $N^{\circ} 27658$ tiene como finalidad primordial mejorar la gestión pública y construir un Estado democrático, descentralizado y al servicio del ciudadano. Dicho proceso busca obtener mayores niveles de eficiencia del aparato estatal, de manera que se logre una mejor atención a la ciudadanía, priorizando y optimizando el uso de los recursos públicos, eficiencia, eficacia y atención que lamentablemente es pésimo.

El objetivo es alcanzar un Estado al servicio de la ciudadanía, con canales efectivos de participación ciudadana, descentralizado y desconcentrado, transparente en su gestión, con servidores públicos calificados y adecuadamente remunerados y fiscalmente equilibrado.

La Ley Orgánica del Poder Ejecutivo $N^{\circ} 29158$, recoge entre sus principios el del servicio al ciudadano, el de inclusión y equidad y el de participación y transparencia. Estos principios procuran que las entidades del Poder Ejecutivo estén al servicio de las personas y de la sociedad y actúen en función de sus necesidades, así como del interés general de la nación, asegurando que su actividad se realice con eficacia, eficiencia, simplicidad, inclusión, equidad, transparencia y participación, de manera que los ciudadanos tengan acceso a la información sobre la gestión del Estado.

\section{REFERENCIAS BIBLIOGRÁFICAS}

Aguilar, L. (2006). Tecnologías de información y Nueva Gestión Pública: Experiencias de gobierno electrónico en México.1ra ed. México: Editores e Impresores FOC.

Andía, W. (2005). Gestión pública: Pautas para la aplicación de los sistemas administrativos. 1ra ed. Perú: Edición y Dirección Editorial.

Banco Mundial. (2013). Se refiere al Gobierno Electrónico como: al uso por las agencias del gobierno de las tecnologías de información (tales como redes WAN, el Internet, y computadoras móviles) que tienen la capacidad de transformar las relaciones con los ciudadanos, las empresas, y con el propio gobierno. Recuperado de http://www.ongei.gob.pe/ quienes/conceptos/10.htm

Benavides, C. (2014). El discurso de participación ciudadana en el marco diseño de la política de gobierno electrónico de la municipalidad distrital de Miraflores. (Tesis para optar el título profesional en licenciada). Pontificia Universidad Católica del Perú. Perú.

Brys, C. (2005). Plan Estratégico para el Gobierno Electrónico de la Provincia de Misiones.
(Tesis con el que optó el grado de Maestro en Informática y Computación). Universidad Nacional de Misiones. Argentina.

Canales, J. (2011). Gobernabilidad y gestión pública. 2da. ed. Madrid: Prentice Hall

Carrasco, S. (2009).Metodología de la Investigación Científica. 2da. ed. Lima: Editorial San Marcos.

Carrillo, E. (2006). La Nueva Administración Pública. 1ra. ed. Madrid: Alianza Editorial.

Criado, F. \& Ramilo, D. (2001) Conceptos generales en el marco de gobierno electrónico. Recuperado de http:// www.ongei.gob.pe/quienes/ conceptos/08.htm.

Dalf, R. (2006). La experiencia del liderazgo. 1ra. ed. España: Thomson International

Delpiazzo, E. (2009), “Del Derecho de la Información al Derecho Informático: Propuesta de Sistematización". $3^{\circ}$ ed. Buenos Aires: Depalma

Faya, P. (2001). E-Goverrerrrent, literature review. Management Priorities and Senior Personnel Secretariat. (Tesis doctoral en Gestión). Universidad de Carleton. Canada.

Fernández, H. (2001). Internet: Su Problemática Jurídica. Buenos Aires: Ediciones Depalma.

Gartner Group, (2000). “E-goverment.... es una innovación continua de los servicios, la participación de los ciudadanos y la forma de gobernar 
mediante la transformación de las relaciones externas e internas a través de la tecnología, el Internet y los nuevos medios de comunicación". Recuperado de http: / / www.ongei.gob.pe/ quienes/conceptos/09.htm

Goin, J. (2001). Desarrollo e implementación de un sistema de trámite documentario para mejorar la gestión documentaria de la municipalidad distrital de Nuevo Chimbote. (Tesis para optar el título de magister en educación en mención en gestión). Universidad Cesar Vallejo. Perú.

Gómez, G. (2008). Planeación y organización de empresas. México: McGraw-HiIl/ Interamericana Editores S.A.

Gutiérrez, C. (2009). Gobierno electrónico en chile, desafíos, perspectivas y oportunidades. Revista gobierno de Chile. Portal del gobierno chileno. 12(2), 1-12. 1ra. ed. Chile: Editorial Océano Chile
Hernández, R., Fernández C. \& Baptista P. (2014). Metodología de la Investigación. 6ta. ed. México: McGraw-Hill/ Interamericana Editores S.A.

Joo, B. (2004). Análisis y propuesta de gestión pedagógica y administrativa de las TIC's, para construir espacios que generen conocimiento en el colegio Champagnat. (Tesis para optar el título profesional en licenciada). Pontificia Universidad Católica del Perú. Perú.

Marval, W. \& Cumaná, W. (2009). Gobierno electrónico como herramienta de gestión. Tesis de maestría de Gestión Pública. Universidad Oriente de Venezuela.

Naser, A., Concha, G. (2011). El gobierno electrónico en la gestión pública. Santiago de Chile: Ediciones Unidas.

Nunes, P. (2008). Gestión de proyectos. México: Editores S.A.

Pardini, A. (2002). Derecho de
Internet. Buenos Aires: Ediciones La Rocca

Ríos, W. (2002). Internet, Comercio Electrónico \& Telecomunicaciones. Bogotá: Legis.

Rodríguez, G. S. (2004). Gobierno electrónico: Hacia la modernización y transparencia de la gestión pública. Unirevista. 11(3), 1-11. Buenos Aires: Editorial Universitaria de Buenos Aires

Ruelas, A., Pérez, P. (2006). El gobierno electrónico: su estudio y perspectivas de desarrollo. Revista de derecho. 21(3), 1-23. Buenos Aires: Ediciones La Rocca

Sánchez, J. (2008). Gestión pública y governance. México: Instituto de Administración Pública del Estado de México

Sotelo, A. (2006). Un gobierno digital en expansión. México: Editores e Impresores FOC

Tamayo, M. (1998). El proceso de la investigación científica. 3ra. ed. México: Limusa. 